\title{
Leptin decreases the expression of low-density lipoprotein receptor via PCSK9 pathway: linking dyslipidemia with obesity
}

Ying $\mathrm{Du}^{\dagger}$, Sha Li ${ }^{\dagger}$, Chuan-Jue Cui, Yan Zhang, Sheng-Hua Yang and Jian-Jun Li ${ }^{*}$

\begin{abstract}
Background: Previous studies have suggested that people with obesity showed elevated serum levels of leptin as well as lipid dysfunction and proprotein convertase subtilisin/kexin type 9 (PCSK9) played an important role in the regulation of lipid metabolism recently. The aim of this study was to determine if leptin participated in regulating the uptake of low-density lipoproteins (LDL) in hepatocytes via PCSK9.

Methods: HepG2 cells were treated with human recombinant leptin. The impact of leptin on cellular low density lipoprotein receptor (LDLR) and PCSK9 protein levels was determined by Western blot. Dil-LDL uptake assay was performed to examine the LDLR function. Specific small interfering RNAs (siRNAs) were used to interfere the expressions of target proteins.

Results: The expression of LDLR and LDL uptake could be significantly down-regulated by leptin treatment while the expressions of PCSK9 and hepatocyte nuclear factor 1a (HNF1a) were enhanced in HepG2 cells. Furthermore, inhibition of PCSK9 or HNF1 a expression by siRNAs rescued the reduction of LDLR expression and LDL uptake by leptin. We found that leptin activated the p38 mitogen-activated protein kinase (p38MAPK) signaling pathway. Moreover, the changes of the expressions of HNF1a, PCSK9, LDLR, and LDL uptake induced by leptin could be blocked by p38MAPK inhibitor (SB203580). Additionally, leptin attenuated the up-regulation of LDLR caused by atorvastatin in HepG2 cells.
\end{abstract}

Conclusions: These findings indicated firstly that leptin reduced LDLR levels in hepatocyte via PCSK9 pathway, suggesting that PCSK9 might be a alternative target for dyslipidemia in the obesity.

Keywords: Leptin, PCSK9, LDLR

\section{Background}

Obesity is one of the most serious chronic diseases worldwide represented by an excess of body fat [1]. A number of previous studies indicated that obesity increased risk for atherosclerotic cardiovascular disease (ASCVD) [2, 3]. Dyslipidemia is the earliest risk factor of ASCVD in obesity [4] which includes the elevated serum levels of low density lipoprotein cholesterol (LDL-C) [5]. It has been demonstrated that elevated LDL-C plays a

\footnotetext{
*Correspondence: lijianjun938@126.com

†Ying Du and Sha Li contributed equally to this work

State Key Laboratory of Cardiovascular Disease, Division of Dyslipidemia,

Fu Wai Hospital, National Center for Cardiovascular Diseases, Chinese

Academy of Medical Sciences and Peking Union Medical College,

XiCheng District, Beijing 100037, China
}

major role in the initiation and development of atherosclerosis in patients with overweight and obesity [5-7]. Unfortunately, the underlying mechanism responsible for elevated LDL-C levels in obesity is not fully understood.

As well established, low density lipoprotein receptors (LDLRs) in the surface of hepatocyte membranes function as critical regulators for circulating LDL-C homeostasis mainly through controlling the rate of liver uptake and the clearance of LDL particles [8, 9]. Several physiological factors which could affect the hepatic LDLR level are recognized. For instance, resistin, which was increased in both circulation and adipose-tissue adipocytes in obesity $[10,11]$, could reduce LDLR expression in human hepatocytes mediated in part by proprotein convertase subtilisin-kexin type 9 (PCSK9) [12]. In fact, 
PCSK9 is a secreted protein which plays a key role in regulation of LDL-C level in the circulation by binding directly to the hepatic LDLRs and blocking their recycling via promoting its degradation in lysosome [13]. So far, there are two best-described trans activator of PCSK9 gene expression in hepatocytes, sterol response element binding proteins (SREBP) [14] and hepatocyte nuclear factor 1 alpha $(H N F 1 \alpha)[15]$.

Besides resistin, leptin is another increased physiological factor in obesity [16] which also comes from adipose tissue [17]. The major function of leptin is that it decreases food intake and increase energy consumption by acting on specific hypothalamic nuclei CART and NPY [18]. Recently, studies in vitro and in vivo have suggested that leptin could also be involved in the pathophysiology of atherosclerosis [19-21]. Furthermore, clinical data have shown the plasma leptin level as an independent predictor for CVD incident [22]. As such, we hypothesized that hyperleptinemia might be another potential mechanism linking obesity to ASCVD and leptin could regulate the expression of LDLR in the liver just like resistin. Leptin has been reported to stimulate p38 mitogen-activated protein kinase (p38MAPK) pathway in different cell types [23-25] and the p38MAPK pathway also participated in the regulation of physiological processes associated with atherosclerosis $[25,26]$. Therefore, we supposed that whether $\mathrm{p} 38 \mathrm{MAPK}$ pathway could play a role in the regulation of PCSK9 and LDLR by leptin. In addition, statins are the major class of drugs to treatment patients with increased serum LDL-C by upregulating the LDLR expression in hepatocytes [12]. It might be, therefore, interesting to investigate whether leptin could diminish the enhanced LDLR expression induced by atorvastatin treatment. These results will help us to find out whether leptin could affect the statin-induced increase of LDLRs.

In this study, the major goal was to determine if human leptin played a role in regulating the uptake of LDL by PCSK9 in hepG2 cells for the sake of exploring the potential links of leptin with PCSK9 in obesity.

\section{Methods}

\section{Cell culture and treatment}

The human hepatoma cell line HepG2 was purchased from Cell Resource Center, IBMS, CAMS/PUMC. HepG2 cells were cultured in $10 \%$ fetal bovine serum (FBS) (Gibco)containing DMEM (Gibco) supplemented with $1 \%$ NEAA (Life technologies), $1 \%$ penicillin- streptomycin at $37^{\circ} \mathrm{C}$, $5 \%(\mathrm{v} / \mathrm{v}) \mathrm{CO}_{2}$. HepG2 cells were treated with human recombinant leptin protein (R\&D Systems, Minneapolis, $\mathrm{MN}, \mathrm{USA})$ at various doses $(0,5,25,50,100$ and $200 \mathrm{ng} /$ $\mathrm{ml}$ ) for $24 \mathrm{~h}$ or with $50 \mathrm{ng} / \mathrm{ml}$ leptin for various times $(0$, $6,12,24,48 \mathrm{~h})$. In other experiments, the p38MAPK
inhibitor-SB203580, was administered at $10 \mu \mathrm{M}$, either alone or combined with $50 \mathrm{ng} / \mathrm{ml}$ leptin for $24 \mathrm{~h}$. Atrovastatin (Sigma, MO) was administered at $10 \mu \mathrm{M}$, either alone or combined with $50 \mathrm{ng} / \mathrm{ml}$ leptin for $24 \mathrm{~h}$.

\section{Western blots}

Cultured cells were collected with cell lysis buffer (Beyotime, Shanghai, China). Protein samples were separated by precast NuPAGE Novex 4-12\% (w/v) Bis-Tris gels (Life technologies, Carlsbad, CA, USA) and then transferred onto nitrocellulose membrane using the iBlotTM dry blotting system as described by the manufacturer (Invitrogen, Carlsbad, CA, USA). Membranes were blocked in TBST buffer (20 mM Tris, pH 7.5, $150 \mathrm{mM}$ $\mathrm{NaCl}, 0.1 \%$ tween 20 ) containing $5 \%$ milk for $1 \mathrm{~h}$ at room temperature and incubated with primary antibodies specific for LDLR (Biovision, Mountain View, CA), PCSK9 (Cayman Chemicals, MI), HNF1 $\alpha$ (Cell Signaling) and GAPDH (Abcam) overnight at $4{ }^{\circ} \mathrm{C}$, and then incubated with a secondary antibody conjugated with horseradish peroxidase (HRP) for $2 \mathrm{~h}$ at room temperature. Blots were developed using chemoluminescence (ECL, Thermo Fisher Scientific, Waltham, MA, USA) on FluorChem M image system.

\section{Dil-LDL uptake assay}

HepG2 cells were changed to serum-free media and incubated with $10 \mu \mathrm{g} / \mathrm{ml}$ DiI-LDL (lifetech) for $24 \mathrm{~h}$ at $37^{\circ} \mathrm{C}$ in the dark. After incubation, the cells were washed with PBS and fixed in the presence of a $4 \%$ paraformaldehyde, and the nuclei were subsequently stained with Hoechst dye. Finally, the cells were examined with fluorescence microcopy (DMI-4000B, Leica).

\section{Small interfering ribonucleic acid (siRNA) transfection} PCSK9 and HNF1 $\alpha$ Stealth siRNA duplexes are followings: PCSK9 Stealth siRNA duplexes (Life tech, Carlsbad, CA, USA) targeting sequences: $5^{\prime}$-GAC AUC AUU GGU GCC UCC AGC GAC U-3' and $5^{\prime}$-AGU CGC UGG AGG CAC CAA UGA UGU C- $3^{\prime}$ and HNF1 $\alpha$ Stealth siRNA duplexes (Life tech, Carlsbad, CA, USA) targeting sequences: $5^{\prime}$-UCG AUA CCA CUG GCC UCA ATT-3' and 5'-UUG AGG CCA GUG GUA UCG ATT-3'. The negative controls siRNA Duplexes (Life tech, Carlsbad, CA, USA) were used as a control. siRNA were transfected into HepG2 cells using Lipofectamine TM RNAiMAX (Life tech, Carlsbad, CA, USA) according to manufacturer's protocol.

\section{Statistical analysis}

Results were presented as mean \pm SEM at least three independent experiments. Significant differences between control and treatment groups were assessed by 
One-way ANOVA with proper posttest or Student twotailed $t$ test.

\section{Results \\ Leptin down-regulates expression of LDLR and up-regulates expression of PCSK9}

To investigate the effect of leptin on the expression of LDLR and PCSK9, we treated HepG2 cells with different concentrations of leptin $(0,5,25,50,100$ and $200 \mathrm{ng} /$ $\mathrm{ml}$ ) for $24 \mathrm{~h}$. As a result, the LDLR protein levels were decreased and the PCSK9 protein levels were increased by leptin stimulation in a dose-dependent manner (Fig. 1a, b). Significant changes in LDLR and PCSK9 protein expression, compared with vehicle-treated cells, were observed at 50,100, $200 \mathrm{ng} / \mathrm{ml}$ of leptin treatments. Subsequently, we used $50 \mathrm{ng} / \mathrm{ml}$ of leptin to stimulate HepG2 cells with different times $(0,6,12,24,48 \mathrm{~h})$. The impact of leptin on LDLR and PCSK9 appeared in a timedependent manner (Fig. 1c, d). The results showed that the PCSK9 expression was significantly enhanced by leptin treatment after $12 \mathrm{~h}$ and the LDLR expression was significantly reduced after $24 \mathrm{~h}$.
PCSK9 inhibition blocks the effect of leptin on LDLR expression and LDL uptake

We further investigated whether the down-regulation of LDLR by leptin was mediated by PCSK9. HepG2 cells were pre-treated with with negative control siRNAs (40 nM), which did not target any gene, or the PCSK9 siRNA for $24 \mathrm{~h}$ before the treatment of leptin. Our results showed that inhibition of endogenous PCSK9 expression using the PCSK9 siRNA (40 nM) abrogated the suppression of the LDLR expression (Fig. 2a, b) and LDL uptake (Fig. 2c) by leptin after $24 \mathrm{~h}$ treatment.

\section{HNF1a inhibition blocks the effect of leptin on LDLR expression and LDL uptake}

HNF1 $\alpha$ is an upstream regulator of PCSK9 which can bind to the promoter of PCSK9 and directly regulate PCSK9 expression [15]. To further confirm that leptin promotes PCSK9 expression by the activation of HNF1 $\alpha$, we transfected HepG2 cells with HNF1 $\alpha$ siRNA for $24 \mathrm{~h}$ before leptin treatment. The data suggested that inhibition of endogenous HNF1 $\alpha$ expression by the HNF1 $\alpha$ siRNA (40 $\mathrm{nM})$ could also abrogate the decrease in LDLR
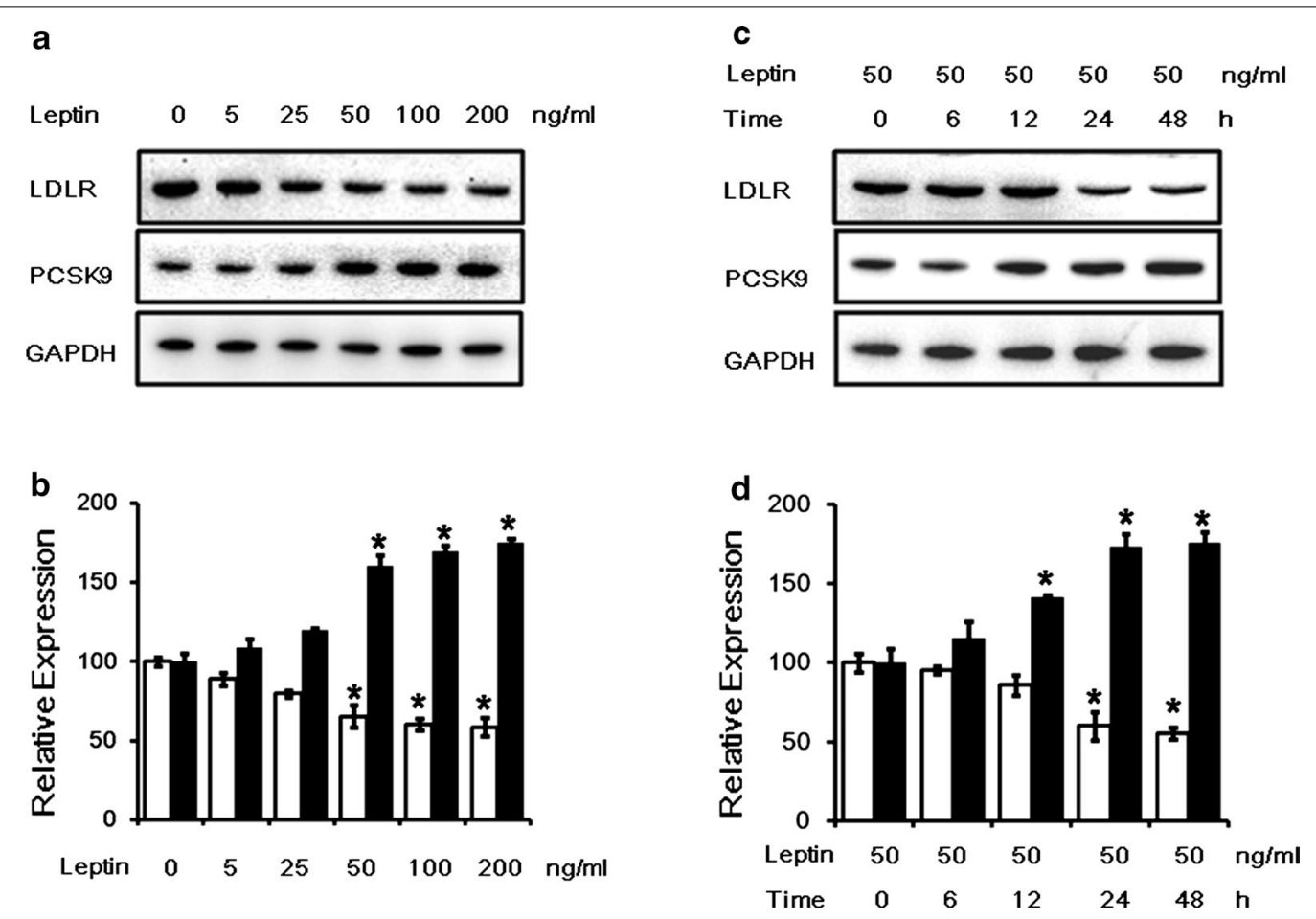

口 LDLR

PCSK9

Fig. 1 The effects of leptin on LDLR and PCSK9 protein levels in HepG2 cells. a Western blot analysis of leptin on LDLR and PCSK9 protein levels in HepG2 cells treated with leptin $(0,5,25,50,100$ and $\mathrm{ng} / \mathrm{mL})$ for $24 \mathrm{~h}$. bThe normalized intensities of LDLR and PCSK9 versus GAPDH are presented as the mean \pm SD of three independent dose-dependent experiments. c Western blot analysis of leptin (50 ng/ml) on LDLR and PCSK9 protein levels in HepG2 cells treated with leptin for 0, 6, 12, 24, 48 h. d The normalized intensities of LDLR and PCSK9 versus GAPDH are presented as the mean \pm SD of three independent time-dependent experiments. ${ }^{*} p<0.05$ represent significant differences compared to the vehicle-treated cells 


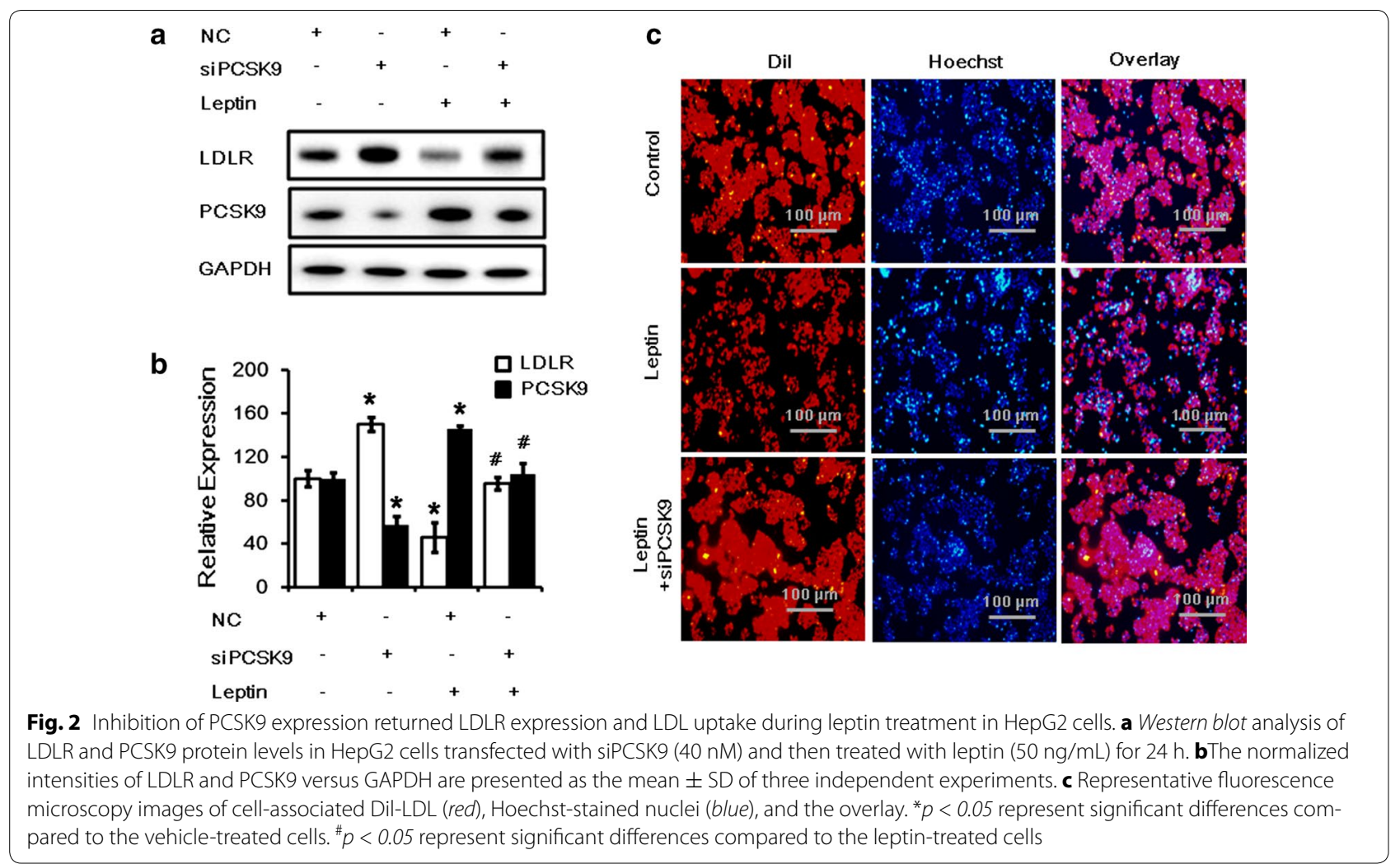

expression and increase in PCSK9 expression (Fig. 3a, b) as well as the suppression of LDL uptake (Fig. 3c) by leptin after $24 \mathrm{~h}$ treatment.

\section{Leptin down-regulates LDLR and up-regulates PCSK9 through p38MAPK pathway}

The MAPK signaling cascades have been shown to be induced by leptin treatment in different cells [23-25]. In this study, we found that phosphorylation of p38 could be induced by leptin in HepG2 cells in a timedependent manner (Fig. 4a, b). To investigate whether p38MAPK pathway was involved in the regulation of LDLR by leptin, HepG2 cells were co-cultured with the p38MAPK specific inhibitor-SB203580 (10 $\mu \mathrm{M})$ alone or combined with leptin. The data indicated that inhibition of the p38MAPK pathway abolished the decrease in LDLR expression and the increase in PCSK9 and HNF1 $\alpha$ expression (Fig. 4c, d) as well as the suppression of LDL uptake (Fig. 4e) by leptin after $24 \mathrm{~h}$ treatment.

\section{Leptin inhibits the up-regulation of LDLR mediated by atorvastatin}

Additionally, we tested whether leptin inhibited the normal statin-mediated up-regulation of hepatic LDLR levels. HepG2 cells were treated with atrovastatin $(10 \mu \mathrm{M})$ alone or combined with leptin $(50 \mathrm{ng} / \mathrm{ml})$ for $24 \mathrm{~h}$.
Consistent with previous studies [12], our results showed that leptin diminished the increase in hepatic LDLR expression induced by atorvastatin treatment (Fig. 5a, b) and increase in cellular PCSK9 protein compared with atorvastatin treatment alone (Fig. $5 \mathrm{c}, \mathrm{d}$ ).

\section{Discussion}

In this study, we, for the first time, demonstrated that leptin significantly increased PCSK9 expression and suppressed LDLR in HepG2 cells. The major novel findings of the present study were as follows: (1) leptin could suppress LDLR level, LDL uptake and elevate PCSK9 expression; (2) the changes of LDLR, PCSK9 levels and LDL uptake were regulated by enhanced expression in HNF1 $\alpha$ and the elevated activation of p38MAPK pathway; (3) leptin could abolish the statin-induced up-regulation of hepatic LDLR level. This study revealed a novel role of leptin in the regulation of hepatic LDLR and PCSK9 expression, which might provide additional information to understand the mechanism responsible for dyslipidemia in obesity.

It is well known that leptin is an adipose tissue-derived hormone, which plasma level is positive correlated with the body fat mass $[16,17]$. The major function of leptin is to regulate human body weight and energy homeostasis by the brain [18]. Considering the roles of leptin in 

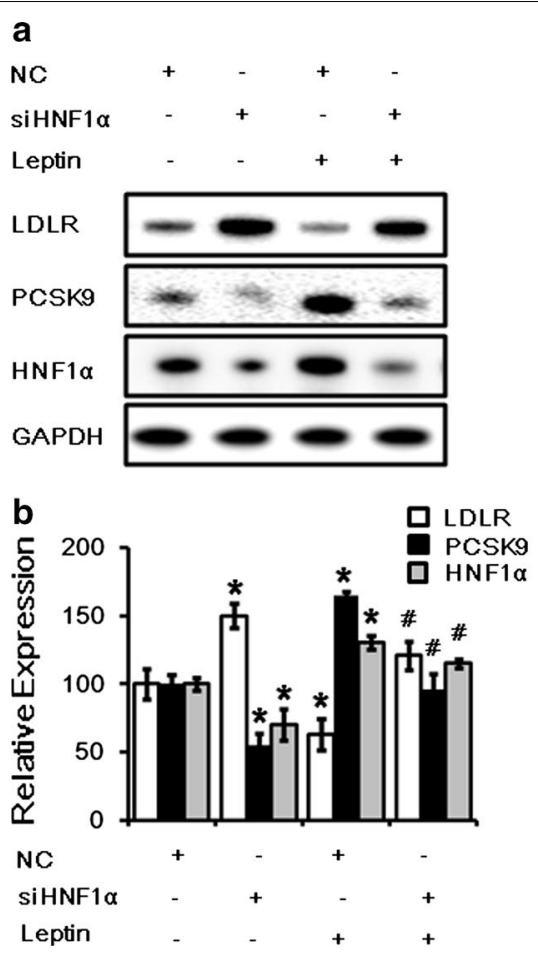

C
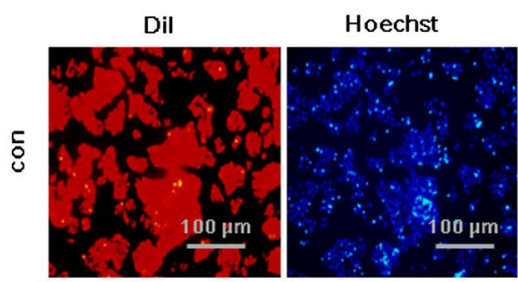

Overlay
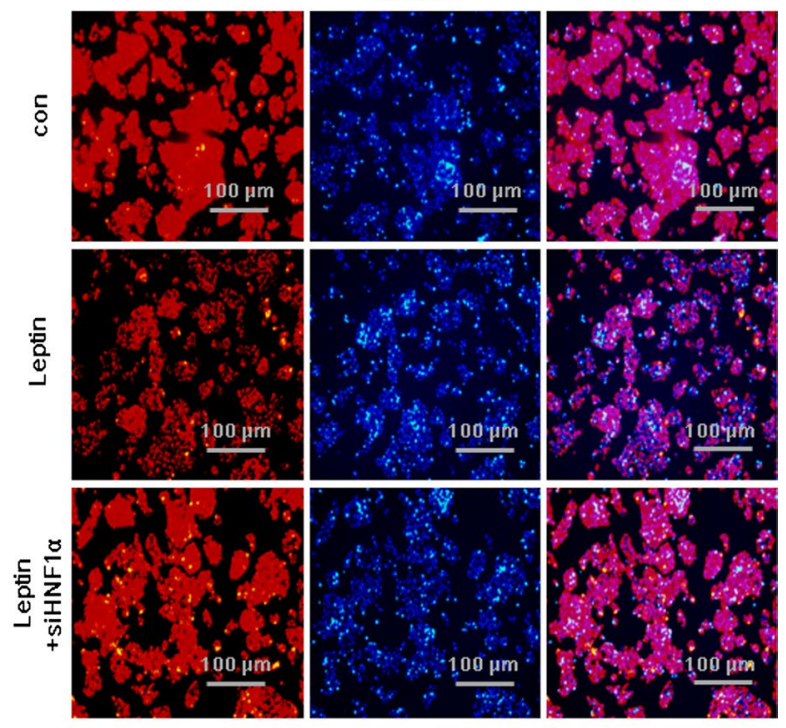

Fig. 3 Inhibition of HNF1 a expression returned LDLR and PCSK9 expression and LDL uptake during leptin treatment in HepG2 cells. a Western blot analysis of LDLR, PCSK9 and HNF1 a protein levels in HepG2 cells transfected with siHNF1a (40 nM) and then treated with leptin (50 ng/mL) for $24 \mathrm{~h}$. b The normalized intensities of LDLR, PCSK9 and HNF1 a versus GAPDH are presented as the mean \pm SD of three independent experiments. $\mathbf{c}$ Representative fluorescence microscopy images of cell-associated Dil-LDL (red), Hoechst-stained nuclei (blue), and the overlay. ${ }^{*} p<0.05$ represent significant differences compared to the vehicle-treated cells. ${ }^{\#} p<0.05$ represent significant differences compared to the leptin-treated cells

physiological processes associated with cardiovascular disease, such as blood pressure [27], platelet aggregation [28], arterial thrombosis [29], angiogenesis [30], and inflammatory vascular responses [31], it, logically, may have a close relationship with the development of CVD [32]. Interestingly, study from Kwakernaak et al. found that plasma leptin had a positive association with plasma PCSK9 in healthy people [33]. Their results suggested that leptin might be involved in the regulation of hepatic PCSK9 expression. Therefore, illuminating the relationship of leptin and PCSK9 in vitro is the major goal of this study.

In the beginning of this study, we found that leptin could cause the decrease of LDLR expression and LDL uptake (Additional file 1: Figure S1) in HepG2 cells. Then, we sought to illuminate the mechanisms by which leptin regulates the hepatic LDLR levels. Definitely, PCSK9 have been reported to regulate LDL levels by mediating LDLR protein degradation [13]. That is, PCSK9 could bind to the extracellular domain of the LDLRs and then lead LDLRs to lysosome-mediated degradation instead of recycling to the cell surface [13]. Therefore, PCSK9 stood a good chance to mediate the regulation of hepatic LDLR levels. Consisting with our assumption, the present study showed that PCSK9 levels were increased by leptin stimulation. Subsequently, we further investigated the role of PCSK9 in the leptin-mediated reduction in LDLR levels. In our study, inhibition of PCSK9 gene expression were carried out by transfecting PCSK9 siRNA into HepG2 cells. Negative control siRNAs were used as controls, which did not affect expression PCSK9 and LDLR (Additional file 2: Figure S2). And the relative transfection efficiency was shown by using the positive control-GAPDH siRNA and it was great in our experiments (Additional file 3: Figure S3). As a result, we found that the suppression of LDLR expression and LDL uptake by leptin stimulation could be reversed by the inhibition of PCSK9 expression. These findings suggested that leptin could suppress the LDLR expression by increasing hepatic PCSK9 level.

HNF $1 \alpha$ is one of the transcriptional regulation factors which regulates PCSK9 expression by binding to its promoter [15]. To determine whether HNF1 $\alpha$ was involved in the regulation of LDLR and PCSK9 by leptin, HNF $1 \alpha$ siRNAs were used to knockdown its expression. It was found that elevation of PCSK9 expression and the reduction of LDLR expression and function by leptin stimulation could be abolished by the inhibition of HNF1 $\alpha$ 


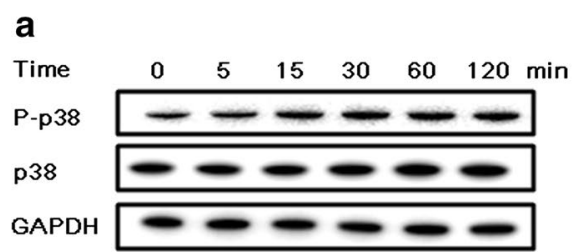

\section{b}
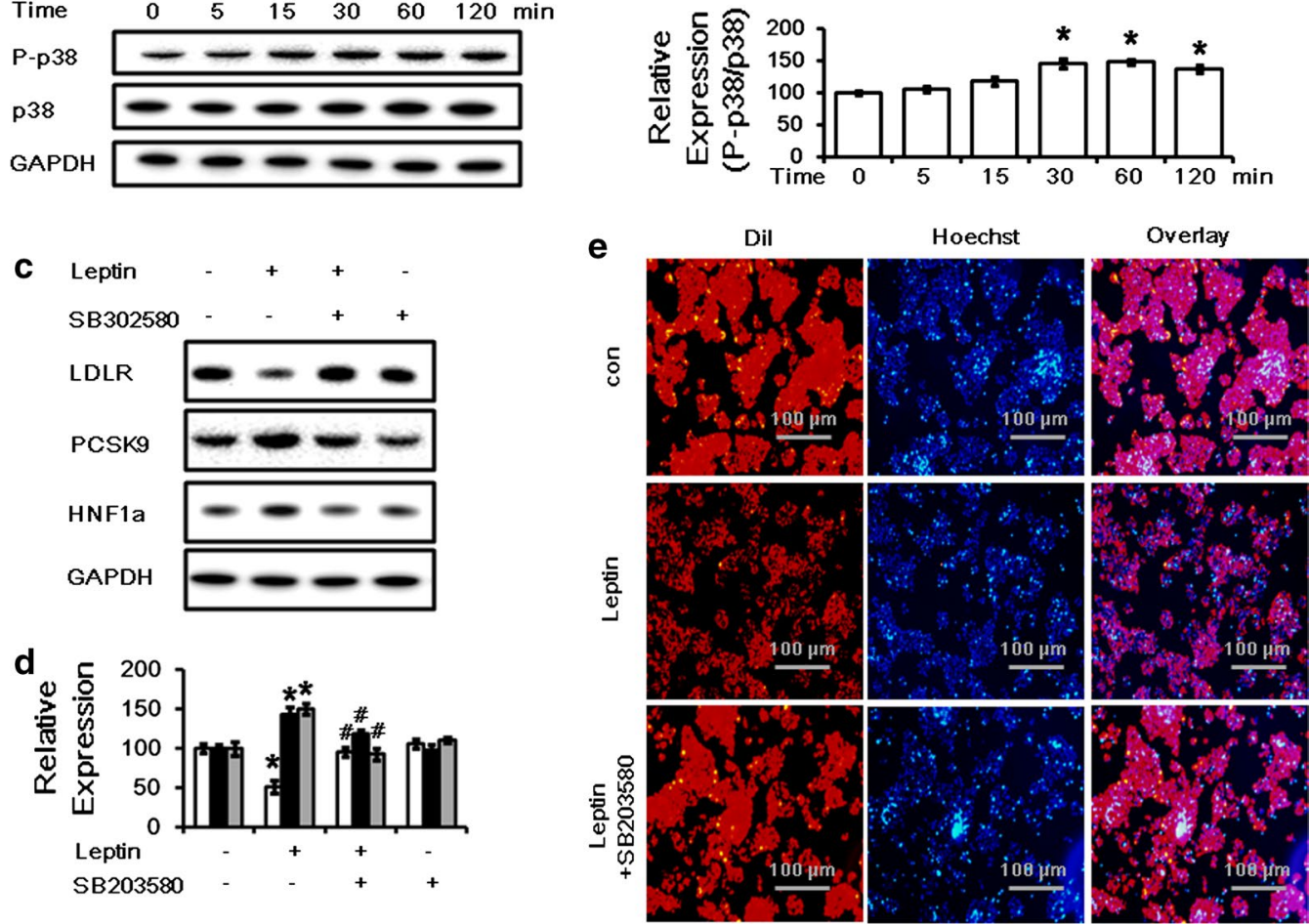

Fig. 4 Leptin modulates LDLR and PCSK9 protein levels and LDL uptake through a p38MAPK-dependent pathway. a Western blot analysis of phosphorylated-p38 and total p38 protein levels in HepG2 cells treated with $50 \mathrm{ng} / \mathrm{ml}$ leptin for 0, 5, 15, 30, 60, $120 \mathrm{~min}$. b The normalized intensities of phosphorylated-p38 versus total p38 are presented as the mean \pm SD of three independent experiments. c Western blot analysis of LDLR, PCSK9 and HNF1a protein levels in HepG2 cells treated with leptin $(50 \mathrm{ng} / \mathrm{mL})$ alone or combined with SB $203580(10 \mu \mathrm{M})$ for $24 \mathrm{~h}$. d The normalized intensities of LDLR, PCSK9 and HNF1a versus GAPDH are presented as the mean \pm SD of three independent experiments. e Representative fluorescence microscopy images of cell-associated Dil-LDL (red), Hoechst-stained nuclei (blue), and the overlay. ${ }^{*} p<0.05$ represent significant differences compared to the vehicle-treated cells. ${ }^{*} p<0.05$ represent significant differences compared to the leptin-treated cells

expression. It is well known that another transcription factor, SREBP2 is the most important trans-activator of PCSK9 which can also induce LDLR expression [14]. Hence, we also examined the SREBP2 expression under leptin treatment for $24 \mathrm{~h}$. Interestingly, we found that there was no significant change of SREBP2 expression by leptin in both mRNA and protein level (Additional file 4: Figure S4 and Additional file 5: Figure S5). Although the exact reason for this unique phenomenon is unclear, we supposed that SREBP2 may not be involved in the regulation of LDLR mediated by PCSK9 under leptin treatment.

Leptin has been reported to signal via various kinases. The activity of p38MAPK pathway is also modulated by leptin in different cell types. For instance, leptin reduced the activity of the $\mathrm{Na}+/ \mathrm{K}+$ ATPase significantly by activating p38MAPK in Caco-2 cells [23]; leptin could inhibit glucose intestinal absorption by activation of p38MAPK [24]; Besides, leptin also could activate human B cells to secrete cytokines via activation of p38MAPK/ ERK1/2 signaling pathways, which may contribute to its inflammatory and immunoregulatory properties [34]. Furthermore, the p38MAPK pathway has also been demonstrated to be participated in the regulation of physiological process associated with atherosclerosis. Activation of p38MAPK pathway could regulate interleukin-16 -induced migration and invasion of vascular smooth muscle cells [26] and be involved in the suppression in oxidized low-density lipoprotein- induced endothelial cell apoptosis by paenonl [25]. Therefore, we examined whether p38MAPK pathway could be activated by leptin treatment. Interestingly, we found that the phosphorylation of p38 could be increased by leptin (Fig. 4a, b). In addition, the inhibition of p38MAPK pathway by its inhibitor SB203580 could abolish the elevation in PCSK9 expression and the suppression of LDLR expression and LDL uptake by leptin stimulation (Fig. 4c, d, e; Additional file 6: Figure S6). On the contrary to our results, Pham DD et al. recently reported that nerve growth factor (NGF) could increase LDLR expression by stimulation of p38MAPK and activation of caspase-3 


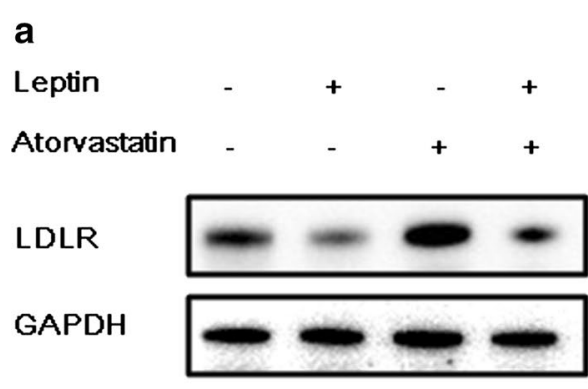

b

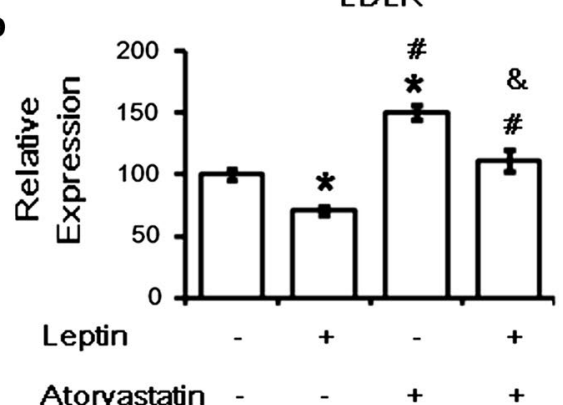

C

Leptin

Atorvastatin

$\begin{array}{llll}- & + & - & + \\ - & - & + & +\end{array}$

PCSK9

GAPDH

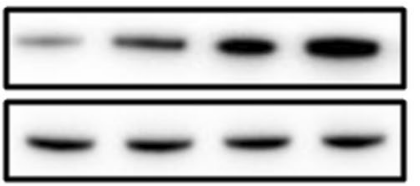

d

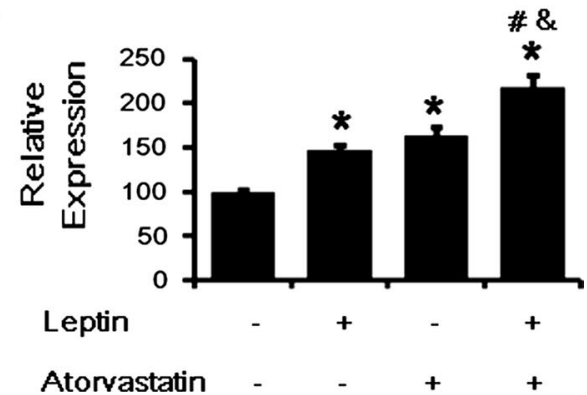

Fig. 5 The effect of a combination of leptin and atrovastatin in HepG2 cells. a Western blot analysis of LDLR protein levels in HepG2 cells treated with leptin $(50 \mathrm{ng} / \mathrm{mL})$ alone or combined with atorastatin $(10 \mu \mathrm{M})$ for $24 \mathrm{~h}$. b The normalized intensity of LDLR versus GAPDH are presented as the mean \pm SD of three independent experiments. c Western blot analysis of PCSK9 protein levels in HepG2 cells treated with leptin (50 ng/ml) alone or combined with atorastatin $(10 \mu \mathrm{M})$ for $24 \mathrm{~h}$. d The normalized intensity of PCSK9 versus GAPDH are presented as the mean \pm SD of three independent experiments. ${ }^{*} p<0.05$ represent significant differences compared to the vehicle-treated cells. ${ }^{*} p<0.05$ represent significant differences compared to the leptin-treated cells. ${ }^{\circledR} p<0.05$ represent significant differences compared to the atorvastatin-treated cells

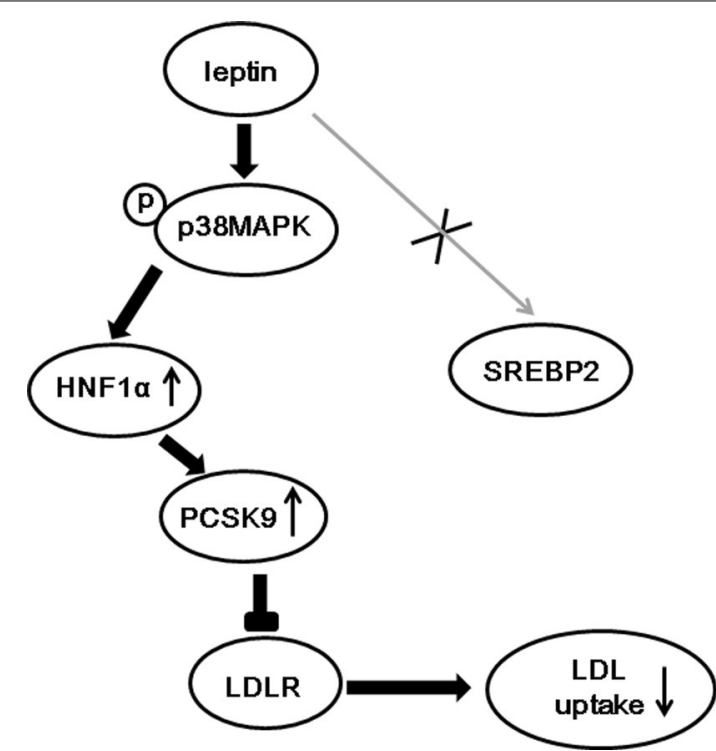

Fig. 6 Summary of pathways by leptin on PCSK9 and LDLR expression in HepG2 cells. Stimulation of p38MAPK pathway by leptin increased expression of nuclear HNF1 a which could bind to PCSK9 promoter, leading to increase PCSK9 expression in HepG2 cells. The increased PCSK9 leaded to elevated LDLR degradation and inhibited LDL uptake and SREBP2 cleavage in hepatocytes [35]. In this study, the activation of SREBP2 was mediated by signaling pathway triggered by ligand-activated p75NTR which were induced by p38MAPK and caspase- 3 . In their study, Caspase-3 activation was caused by p38MAPK following NGF stimulations. In our study, leptin did not change the levels of SREBP2 proteins including both uncleaved and mature form (Additional file 5: Figure S5) but activate the p38MAPK. The possible reason is that leptin could suppress caspase- 3 activity in HepG2 cells [36]. Thereby the p75NTR could not be activated and then SREBP2 cleavage would not be affected by letpin.

Additionally, statins are the major-class therapy currently used for patients with increased serum LDL-C by upregulating the LDLR expression in hepatocytes [12]. Therefore, it might be interesting to investigate whether leptin could diminish the enhanced LDLR expression induced by atorvastatin treatment. Our results showed that leptin could block the increase of LDLR expression induced by atorvastatin treatment (Fig. 5). This inhibition in the statin-induced LDLR up-regulation by leptin was partially contributed by increasing PCSK9 protein level. These results suggested that inhibitors for leptin might enhance hepatic LDLR expression and reduce plasma 
LDL-C level when it was administered combined with statins. Further studies are needed to conform our findings and test our hypothesis in the future.

\section{Conclusion}

In conclusion, leptin could down-regulate the expression and function of LDLR and up-regulate the expression of PCSK9 through the p38MAPK- and HNF1 $\alpha$-dependent mechanism (Fig. 6). Our findings supported that the regulation of LDLR and PCSK9 by leptin might be potential mechanism linking obesity to ASCVD.

\section{Additional files}

Additional file 1: Figure S1. Time course of LDL uptake response to leptin treatment in HepG2 cells. Representative fluorescence microscopy images of cell-associated Dil-LDL (red), Hoechst-stained nuclei (blue), and the overlay in HepG2 cells treated with leptin (50 ng/ml) for 0, 6, 12, 24, $48 \mathrm{~h}$.

Additional file 2: Figure S2. Effect of negative control siRNA on LDLR and PCSK9 expression. Real time PCR analysis of LDLR and PCSK9 mRNA levels in HepG2 cells transfected with negative control siRNA for 24 h. Con, control (vehicle); NC, negative control.

Additional file 3: Figure S3. Knockdown efficiency of GAPDH siRNA in HepG2 cells. Real time PCR analysis of GAPDH mRNA levels in HepG2 cells transfected with GAPDH siRNA for $24 \mathrm{~h}$. ${ }^{*} p<0.05$ represent significant differences compared to the vehicle-treated cells.

Additional file 4: Figure S4. Examination of the effect of leptin on PCSK9, LDLR, HNF1 a and SREBP2 mRNA Levels in dose- or time-dependent manner in HepG2 cells. (A) Real time PCR analysis of LDLR, PCSK9, HNF1 $a$ and SREBP2 mRNA levels in HepG2 cells treated with leptin (0, $5,25,50,100$ and $\mathrm{ng} / \mathrm{mL}$ ) for $24 \mathrm{~h}$. (B) Real time analysis of LDLR, PCSK9, HNF1 1 and SREBP2 mRNA levels in HepG2 cells treated with leptin (50 ng/ $\mathrm{ml}$ ) for $0,6,12,24,48 \mathrm{~h}$. ${ }^{*} p<0.05$ represent significant differences expression compared to the vehicle-treated cells.

Additional file 5: Figure S5. Effect of leptin on expression of SREBP2. (A) Western blot analysis of SREBP2 protein levels in HepG2 cells treated with leptin $(50 \mathrm{ng} / \mathrm{mL}$ ) for $24 \mathrm{~h}$. (B) The normalized intensities of uncleaved and mature SREBP2 versus GAPDH are presented as the mean \pm SD of three independent experiments.

Additional file 6: Figure S6. Effect of p38MAPK inhibitor with different concentrations on LDLR and PCSK9 expression. (A) Western blot analysis of LDLR and PCSK9 protein levels in HepG2 cells treated with leptin $(50 \mathrm{ng} / \mathrm{mL})$ alone with SB203580 (1 $\mu \mathrm{M}$ or $10 \mu \mathrm{M})$ for $24 \mathrm{~h}$. (B) The normalized intensities of LDLR and PCSK9 versus GAPDH are presented as the mean \pm SD of three independent experiments. ${ }^{*} p<0.05$ represent significant differences compared to the vehicle- treated cells. ${ }^{\#} p<0.05$ represent significant differences compared to the leptin-treated cells.

\section{Abbreviations}

ASCVD: atherosclerotic cardiovascular disease; HepG2: human hepatocarcinoma cell line 2; HNF1a: hepatocyte nuclear factor 1 alpha; LDL: Iow density lipoprotein; LDLR: low density lipoprotein receptor; MAPK: mitogen-activated protein kinase; PCSK9: proprotein convertase subtilisin-kexin type 9; SREBP: sterol response element binding proteins.

\section{Authors' contributions}

DY and LS completed the project, and analyzed the data, and wrote the manuscript. LJJ established the study, interpreted the data, and contributed to reviewed/edited the manuscript. The other co-authors for this manuscript contributed to collect the data and conduct the experiments. We thank the staff and participants of this study for their important contributions. All authors read and approved the final manuscript.

\section{Acknowledgements}

This work was partially supported by the National Natural Science Foundation of China $(81070171,81241121)$, the Specialized Research Fund for the Doctoral Program of Higher Education of China (20111106110013), the Capital Special Foundation of Clinical Application Research (Z121107001012015), the Capital Health Development Fund (2011400302, 2016-1-4035), and the Beijing Natural Science Foundation (7131014) awarded to Dr. Jian-Jun Li, MD, PhD.

\section{Competing interests}

The authors declare that they have no competing interests.

\section{Availability of data and materials}

The relevant raw data will not be shared because the data from cell described and presented in the manuscript are completed and clear for testing by reviewers.

Received: 16 May 2016 Accepted: 8 September 2016

Published online: 23 September 2016

\section{References}

1. Sainz N, Barrenetxe J, Moreno-Aliaga MJ, Martinez JA. Leptin resistance and diet-induced obesity: central and peripheral actions of leptin. Metabolism. 2015;64(1):35-46.

2. Grundy SM. Obesity, metabolic syndrome, and cardiovascular disease. J Clin Endocrinol Metab. 2004;89(6):2595-600.

3. Despres JP, Lemieux I. Abdominal obesity and metabolic syndrome. Nature. 2006:444(7121):881-7.

4. Fox CS, Massaro JM, Hoffmann U, Pou KM, Maurovich-Horvat P, Liu CY, Vasan RS, Murabito JM, Meigs JB, Cupples LA, et al. Abdominal visceral and subcutaneous adipose tissue compartments: association with metabolic risk factors in the Framingham Heart Study. Circulation. 2007;116(1):39-48.

5. Superko HR, Gadesam RR. Is it LDL particle size or number that correlates with risk for cardiovascular disease? Curr Atheroscler Rep. 2008;10(5):377-85.

6. Rosenson RS. Low high-density lipoprotein cholesterol disorders and cardiovascular risk: contribution of associated low-density lipoprotein subclass abnormalities. Curr Opin Cardiol. 2005:20(4):313-7.

7. Rosenson RS. Colesevelam HCI reduces LDL particle number and increases LDL size in hypercholesterolemia. Atherosclerosis. 2006;185(2):327-30.

8. Chan DC, Watts GF, Redgrave TG, Mori TA, Barrett PH. Apolipoprotein B-100 kinetics in visceral obesity: associations with plasma apolipoprotein C-III concentration. Metabolism. 2002;51(8):1041-6.

9. Attie $A D$, Seidah NG. Dual regulation of the $L D L$ receptor-some clarity and new questions. Cell Metab. 2005;1(5):290-2.

10. Degawa-Yamauchi M, Bovenkerk JE, Juliar BE, Watson W, Kerr K, Jones R, Zhu Q, Considine RV. Serum resistin (FIZZ3) protein is increased in obese humans. J Clin Endocrinol Metab. 2003;88(11):5452-5.

11. Piestrzeniewicz K, Luczak K, Komorowski J, Maciejewski M, Jankiewicz Wika J, Goch JH. Resistin increases with obesity and atherosclerotic risk factors in patients with myocardial infarction. Metabolism. 2008:57(4):488-93.

12. Melone M, Wilsie L, Palyha O, Strack A, Rashid S. Discovery of a new role of human resistin in hepatocyte low-density lipoprotein receptor suppression mediated in part by proprotein convertase subtilisin/kexin type 9. J Am Coll Cardiol. 2012;59(19):1697-705.

13. Lambert G. Unravelling the functional significance of PCSK9. Curr Opin Lipidol. 2007;18(3):304-9.

14. Jeong HJ, Lee HS, Kim KS, Kim YK, Yoon D, Park SW. Sterol-dependent regulation of proprotein convertase subtilisin/kexin type 9 expression by sterol-regulatory element binding protein-2. J Lipid Res. 2008;49(2):399-409. 
15. Li H, Dong B, Park SW, Lee HS, Chen W, Liu J. Hepatocyte nuclear factor 1alpha plays a critical role in PCSK9 gene transcription and regulation by the natural hypocholesterolemic compound berberine. J Biol Chem. 2009:284(42):28885-95.

16. Otero M, Lago R, Lago F, Casanueva FF, Dieguez C, Gomez-Reino JJ, Gualillo O. Leptin, from fat to inflammation: old questions and new insights. FEBS Lett. 2005;579(2):295-301.

17. Zhang Y, Proenca R, Maffei M, Barone M, Leopold L, Friedman JM. Positional cloning of the mouse obese gene and its human homologue. Nature. 1994;372(6505):425-32.

18. Arora S. Anubhuti: role of neuropeptides in appetite regulation and obesity-a review. Neuropeptides. 2006;40(6):375-401.

19. Beltowski J. Leptin and atherosclerosis. Atherosclerosis. 2006;189(1):47-60.

20. Guzik TJ, Mangalat D, Korbut R. Adipocytokines-novel link between inflammation and vascular function? J Physiol Pharmacol. 2006;57(4):505-28.

21. Dubey $L$, Hesong Z. Role of leptin in atherogenesis. Exp Clin Cardiol. 2006;11(4):269-75.

22. Wolk R, Berger P, Lennon RJ, Brilakis ES, Johnson BD, Somers VK. Plasma leptin and prognosis in patients with established coronary atherosclerosis. J Am Coll Cardiol. 2004;44(9):1819-24.

23. El-Zein O, Usta J, El Moussawi L, Kreydiyyeh SI. Leptin inhibits the $\mathrm{Na}(+) / \mathrm{K}(+)$ ATPase in Caco-2 cells via PKC and p38MAPK. Cell Signal. 2015;27(3):416-23.

24. El-Zein O, Kreydiyyeh SI. Leptin inhibits glucose intestinal absorption via PKC, p38MAPK, PI3 K and MEK/ERK. PLoS ONE. 2013;8(12):e83360.

25. Bao MH, Zhang YW, Zhou HH. Paeonol suppresses oxidized lowdensity lipoprotein induced endothelial cell apoptosis via activation of LOX-1/p38MAPK/NF-kappaB pathway. J Ethnopharmacol. 2013;146(2):543-51.

26. Park SL, Hwang B, Lee SY, Kim WT, Choi YH, Chang YC, Kim WJ, Moon SK. p21WAF1 Is Required for Interleukin-16-Induced Migration and Invasion of Vascular Smooth Muscle Cells via the p38MAPK/Sp-1/MMP-9 Pathway. PLOS ONE. 2015;10(11):e0142153.
27. Paleczny B, Siennicka A, Zacharski M, Jankowska EA, Ponikowska B, Ponikowski P. Increased body fat is associated with potentiation of blood pressure response to hypoxia in healthy men: relations with insulin and leptin. Clin Auton Res. 2016;26(2):107-16.

28. Sugiyama C, Ishizawa M, Kajita K, Morita H, Uno Y, Matsubara K, Matsumoto $M$, Ikeda T, Ishizuka T. Platelet aggregation in obese and diabetic subjects: association with leptin level. Platelets. 2007;18(2):128-34.

29. Konstantinides S, Schafer K, Koschnick S, Loskutoff DJ. Leptindependent platelet aggregation and arterial thrombosis suggests a mechanism for atherothrombotic disease in obesity. J Clin Investig. 2001:108(10):1533-40.

30. Tahergorabi Z, Khazaei M. Leptin and its cardiovascular effects: focus on angiogenesis. Adv Biomed Res. 2015:4:79.

31. Abella V, Scotece M, Conde J, Lopez V, Lazzaro V, Pino J, Gomez-Reino JJ, Gualillo O. Adipokines, metabolic syndrome and rheumatic diseases. J Immunol Res. 2014;2014:343746.

32. Sattar N, Wannamethee G, Sarwar N, Chernova J, Lawlor DA, Kelly A, Wallace AM, Danesh J, Whincup PH. Leptin and coronary heart disease: prospective study and systematic review. J Am Coll Cardiol. 2009;53(2):167-75.

33. Kwakernaak AJ, Lambert G, Dullaart RP. Relationship of proprotein convertase subtilisin-kexin type 9 levels with resistin in lean and obese subjects. Clin Biochem. 2012;45(16-17):1522-4.

34. Agrawal S, Gollapudi S, Su H, Gupta S. Leptin activates human B cells to secrete TNF-alpha, IL-6, and IL-10 via JAK2/STAT3 and p38MAPK/ERK1/2 signaling pathway. J Clin Immunol. 2011;31(3):472-8.

35. Pham DD, Do HT, Bruelle C, Kukkonen JP, Eriksson O, Mogollon I, Korhonen LT, Arumae U, Lindholm D. p75 neurotrophin receptor signaling activates sterol regulatory element-binding protein-2 in hepatocyte cells via p38 mitogen-activated protein kinase and caspase-3. J Biol Chem. 2016;291(20):10747-58.

36. Nepal S, Kim MJ, Hong JT, Kim SH, Sohn DH, Lee SH, Song K, Choi DY, Lee ES, Park PH. Autophagy induction by leptin contributes to suppression of apoptosis in cancer cells and xenograft model: involvement of p53/ FoxO3A axis. Oncotarget. 2015:6(9):7166-81.

\section{Submit your next manuscript to BioMed Central and we will help you at every step:}

- We accept pre-submission inquiries

- Our selector tool helps you to find the most relevant journal

- We provide round the clock customer support

- Convenient online submission

- Thorough peer review

- Inclusion in PubMed and all major indexing services

- Maximum visibility for your research

Submit your manuscript at www.biomedcentral.com/submit 